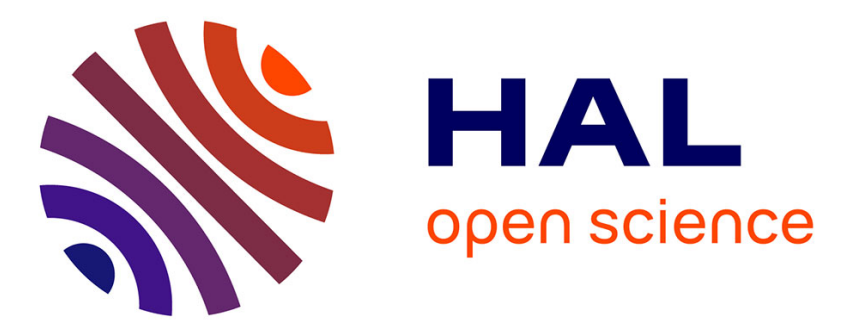

\title{
Longueur de diffusion des porteurs minoritaires et structure de jonction des diodes $\mathrm{Cu} / \mathrm{Cu} 2 \mathrm{O}$
}

\author{
C. Noguet, D. Pierrat, M. Tapiero, J.P. Zielinger
}

\section{To cite this version:}

C. Noguet, D. Pierrat, M. Tapiero, J.P. Zielinger. Longueur de diffusion des porteurs minoritaires et structure de jonction des diodes $\mathrm{Cu} / \mathrm{Cu} 2 \mathrm{O}$. Revue de Physique Appliquée, 1980, 15 (3), pp.595-602. 10.1051/rphysap:01980001503059500 . jpa-00244766

\section{HAL Id: jpa-00244766 https://hal.science/jpa-00244766}

Submitted on 1 Jan 1980

HAL is a multi-disciplinary open access archive for the deposit and dissemination of scientific research documents, whether they are published or not. The documents may come from teaching and research institutions in France or abroad, or from public or private research centers.
L'archive ouverte pluridisciplinaire HAL, est destinée au dépôt et à la diffusion de documents scientifiques de niveau recherche, publiés ou non, émanant des établissements d'enseignement et de recherche français ou étrangers, des laboratoires publics ou privés. 


\title{
Longueur de diffusion des porteurs minoritaires et structure de jonction des diodes $\mathrm{Cu} / \mathrm{Cu}_{2} \mathrm{O}\left({ }^{*}\right)$
}

\author{
C. Noguet, D. Pierrat, M. Tapiero et J. P. Zielinger
}

Laboratoire de Spectroscopie et d'Optique du Corps Solide (**)

Université Louis-Pasteur, 5, rue de l'Université, 67000 Strasbourg, France

(Reçu le 25 juillet 1979, révisé le 19 décembre 1979, accepté le 20 décembre 1979)

\begin{abstract}
Résumé. - La longueur de diffusion $L_{\mathrm{n}}$ des porteurs minoritaires dans des monocristaux de $\mathrm{Cu}_{2} \mathrm{O}$ obtenus principalement par tirage a été mesurée par les méthodes du photovoltage de surface, du spectre photovoltaique et du courant induit par balayage électronique. Dans les deux derniers cas des jonctions $\mathrm{Cu} / \mathrm{Cu}_{2} \mathrm{O}$ ont été utilisées. Les valeurs obtenues par les trois méthodes sont du même ordre de grandeur, elles varient d'un échantillon à l'autre mais ne dépassent pas $8 \mu \mathrm{m}$. Des expériences encore fragmentaires n'ont pu mettre en évidence une relation significative entre longueur de diffusion et résistivité du matériau. Les mesures au microscope électronique révèlent une variation spatiale à courte portée de $L_{\mathrm{n}}$ et une grande inhomogénéité de la collection. La topographie du courant induit montre dans les diodes une zone semi-passive qui peut s'étendre sur une profondeur de quelques $\mu \mathrm{m}$ à partir du métal et qui est interprétée par une forte diminution de la durée de vie des porteurs créés au voisinage de la surface. Cette hypothèse est en accord semi-quantitatif avec l'ordre de grandeur des courants observés et avec les modifications de la réponse spectrale d'une cellule à l'autre. La discussion de ces résultats indique qu'avec $\mathrm{Cu}_{2} \mathrm{O}$ les structures de type Schottky frontales sont inaptes à assurer une conversion photovoltaïque satisfaisante du rayonnement solaire.
\end{abstract}

\begin{abstract}
The diffusion length $L_{\mathrm{n}}$ of minority carriers (electrons) in $\mathrm{Cu}_{2} \mathrm{O}$ single crystals, obtained mainly by pulling, has been measured by the surface photovoltage, the photovoltaic spectrum and the electron beam induced current (EBIC) methods. In the two last cases, $\mathrm{Cu} / \mathrm{Cu}_{2} \mathrm{O}$ junctions have been used. The values for $L_{n}$, obtained by the three methods, are of the same order of magnitude, they differ from sample to sample but lie always below $8 \mu \mathrm{m}$. Preliminary experiments could not reveal any significant correlation between the diffusion length and the resistivity of the material. Measurements by means of the electronic beam reveal a short range variation in space of $L_{\mathrm{n}}$ and a large inhomogeneity of the collection. The topography of the current induced in the diodes by the electron beam shows a semi-passive zone which may extend from the metal to a depth of a few $\mu \mathrm{m}$; this phenomenon is interpreted as resulting from a strong reduction of the minority carrier lifetime near the surface. This assumption agrees, in a semi-quantitative manner, with the order of magnitude of the observed photovoltaic short circuit current and with the shift from cell to cell of the peak in the photovoltaic spectral response. From these results it can be concluded that in the case of $\mathrm{Cu}_{2} \mathrm{O}$, frontwall Schottky barrier type cells are not suitable for an efficient photovoltaic solar energy conversion.
\end{abstract}

1. Introduction. - Il a été souvent indiqué que $\mathrm{Cu}_{2} \mathrm{O}$, semiconducteur de type $\mathrm{p}$ exclusivement, est, de par la largeur de sa bande interdite $(2 \mathrm{eV})$ et de par son coût de production modique, un matériau potentiellement intéressant pour la conversion photovoltaïque de l'énergie solaire. Parmi les différents types de capteurs effectivement réalisés et plus ou moins extensivement étudiés, les structures Schottky sont les plus faciles à réaliser. Les analyses récentes de contacts métal- $\mathrm{Cu}_{2} \mathrm{O}$ par spectroscopie Auger $[1,2]$

$\left(^{*}\right)$ Ce travail a été réalisé, en partie, dans le cadre d'une A.T.P. du Pirdes.

$\left({ }^{* *}\right)$ Associé au C.N.R.S. semblent indiquer l'intervention de phénomènes d'interdiffusion conduisant toujours à des jonctions de type $\mathrm{Cu}-\mathrm{Cu}_{2} \mathrm{O}$. De fait, les hauteurs de barrière mesurées sont très peu sensibles à la nature du métal constituant l'électrode redresseuse [3]. Le présent travail est donc consacré aux cellules $\mathrm{Cu}-\mathrm{Cu}_{2} \mathrm{O}$ de type frontal.

Les cellules $\mathrm{Cu}-\mathrm{Cu}_{2} \mathrm{O}$ réalisées jusqu'ici l'ont été principalement à partir d'un matériau obtenu par oxydation directe de plaquettes de cuivre à haute température et soumis à des traitements thermiques destinés tant à favoriser la croissance cristalline qu'à abaisser la résistivité $[3,4]$. Les performances de la plupart d'entre elles restent très modestes (typi- 
quement $V_{\text {co }} \lesssim 0,4 \mathrm{~V}, J_{\text {cc }} \lesssim 1 \mathrm{~mA} / \mathrm{cm}^{2}, \eta \lesssim 0,2 \%$ ). Exceptionnellement, des rendements de l'ordre de $1 \%$ ont été cités [3].

Deux ensembles relativement disjoints de processus concourent dans le fonctionnement d'une cellule photovoltaique : les processus de transport et les processus de collection. Les premiers sont largement commandés par la diffusion. Pour une jonction donnée, le rendement optimal est obtenu lorsque la longueur de diffusion du porteur minoritaire est suffisante pour adapter le spectre de puissance du flux lumineux incident au spectre d'absorption du matériau. Ce rôle est particulièrement critique avec $\mathrm{Cu}_{2} \mathrm{O}$ où le caractère faiblement permis des transitions directes vers la première bande de conduction conduit à un front d'absorption beaucoup moins abrupt que celui d'autres matériaux, GaAs par exemple [5]. Ce n'est que pour des longueurs de diffusion $L_{\mathrm{n}}$ supérieures à $40 \mu \mathrm{m}$ que plus de $80 \%$ des porteurs créés par un éclairement de type AM1 peuvent être collectés [6]. Avec une valeur mesurée de l'ordre de quelques $\mu \mathrm{m}[4,7]$, la longueur de diffusion s'avère être un des facteurs limitatifs déterminants et mérite à ce titre une étude plus approfondie.

Les méthodes les plus couramment utilisées pour mesurer les faibles longueurs de diffusion sont basées sur le fait que, moyennant certaines conditions, le signal photovoltaïque dépend du coefficient d'absorption $\alpha$ par l'intermédiaire de l'argument

$$
\left(1+\frac{1}{L_{\mathrm{n}} \alpha}\right)^{-1} \quad[16] \text {. }
$$

On en déduit alors $L_{\mathrm{n}}$ soit de la réponse spectrale de l'effet photovoltaïque de surface (PVS) [8,9], soit de la réponse spectrale d'une jonction de type Schottky (SPV) [4].

Ces méthodes, qui sont non destructrices et d'une mise en œuvre relativement aisée, présentent le désavantage de reposer sur le postulat d'une longueur de diffusion unique dans le plan d'illumination comme dans la profondeur du matériau. Lorsqu'il n'en est pas ainsi ou encore lorsque la collection n'est pas uniforme le long de la jonction, les résultats expérimentaux peuvent être d'une interprétation douteuse voire fallacieuse. Il est donc souhaitable de confronter les conclusions déduites de ces mesures aux résultats obtenus par une méthode qui serait spatialement sélective et d'une interprétation plus directe. Ces exigences sont remplies par la méthode de génération de paires électron-trou au moyen du pinceau électronique d'un microscope à balayage et par la mesure du déclin du courant photovoltaïque en fonction de la distance séparant le point d'impact d'une jonction drainant les porteurs minoritaires (EBIC) [10]. Si, de plus, le faisceau d'un tube cathodique est couplé pour son intensité au courant de diode et pour son balayage au balayage du microscope, on visualise sur l'écran la topographie de la contribution des différents points de la surface de l'échantillon. Par ce moyen, on peut en outre obtenir, avec un excellent pouvoir séparateur, des renseignements sur la région de jonction et des informations sur un certain nombre de phénomènes pouvant limiter les processus de collection.

2. Le matériau. - Le matériau qui a servi à nos expériences a été obtenu par tirage à partir d'un bain fondu dans un four à image. Après sciage du barreau, on obtient des flans monocristallins d'environ 8-10 mm de diamètre dont les faces sont sensiblement perpendiculaires à l'axe (110) du cristal. Leur résistivité dépend du barreau d'origine et de leur position dans ce barreau. A température ordinaire, elle est de $5 \times 10^{2}$ à $10^{4} \Omega \mathrm{cm}$ au voisinage du germe et quelque cent fois plus grande à l'autre extrémité [4]. Sa variation thermique présente souvent, aux alentours de la température ordinaire, un palier qui peut s'étendre sur une centaine de degrés et qui est lié vraisemblablement à la saturation de niveaux accepteurs pratiquement compensés (Fig. 1). De tels échantillons sont très sensibles aux recuits sous vide, même à des températures modérées : en augmentant la température du recuit, on fait disparaître peu à peu le palier (Fig. 1) et il ne subsiste plus qu'une anomalie traduisant, au moins en partie, la désexcitation de l'effet de photomémoire [11].

Une statistique effectuée sur une vingtaine de ces

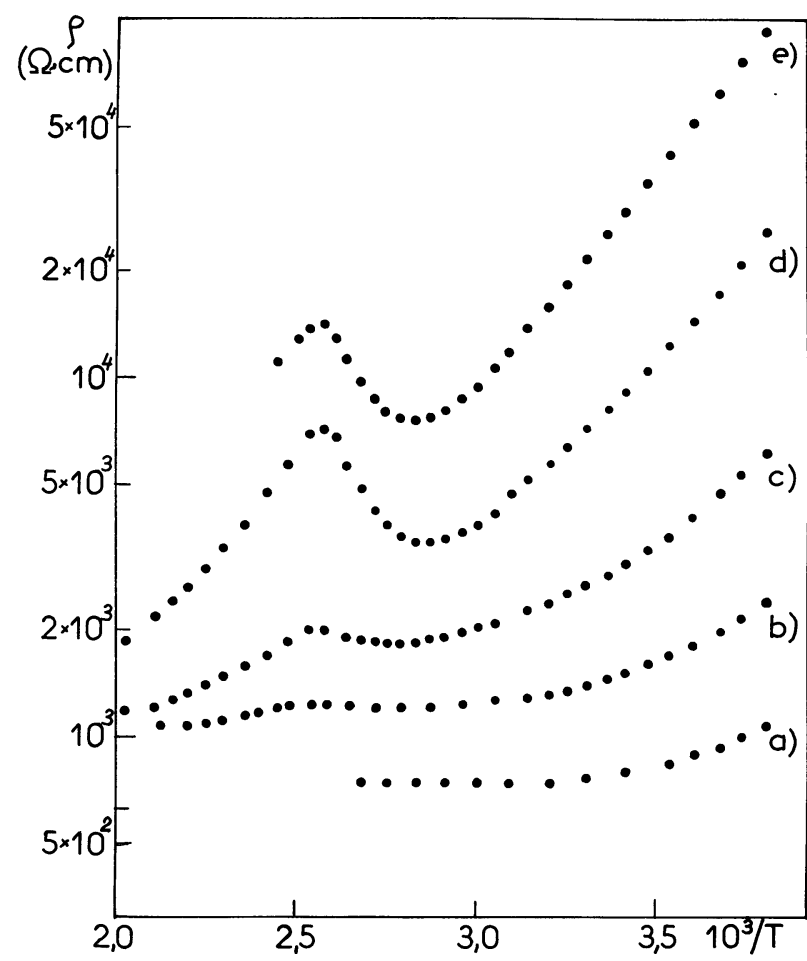

Fig. 1. - Evolution de la variation thermique de la résistivité en fonction de la température maximale $T_{\mathrm{m}}$ du cycle $: a$ ) Etat d'origine ; b) $T_{\mathrm{m}}=140^{\circ} \mathrm{C}$; c) $T_{\mathrm{m}}=195^{\circ} \mathrm{C} ;$ d) $\left.T_{\mathrm{m}}=235^{\circ} \mathrm{C} ; e\right) T_{\mathrm{m}}=280^{\circ} \mathrm{C}$

[Modification of the thermal dependence of the resistivity as a function of the maximum temperature of the cycle : a) Initial state; b) $T_{\mathrm{m}}=140^{\circ} \mathrm{C}$; ) $T_{\mathrm{m}}=195^{\circ} \mathrm{C} ;$ d) $T_{\mathrm{m}}=235^{\circ} \mathrm{C}$; d) $T_{\mathrm{m}}=280^{\circ} \mathrm{C}$.] 
cristaux montre que trois niveaux caractérisés par des énergies d'activation de 0,$18 ; 0,24$ et $0,32 \mathrm{eV}$ en commandent la conductivité, alors que dans le matériau de synthèse, de résistivité plus élevée, l'énergie d'activation peut varier quasi continuement de 0,4 a $1 \mathrm{eV}$ [12]. Des expériences en cours tendent à prouver que ces trois niveaux préexistent dans un même échantillon, mais que certains peuvent être inhibés par une compensation plus ou moins importante liée vraisemblablement à des conditions de refroidissement un peu différentes suivant les tirages.

Les cellules photovoltaïques obtenues par dépôt d'une couche semi-transparente de cuivre (évaporation ou pulvérisation) sur ce matériau ont des performances tout aussi médiocres [4] que celles confectionnées à partir du matériau obtenu par synthèse directe.

Bien que la hauteur de barrière, évaluée tant à partir des caractéristiques $I-V$ qu'à partir de l'effet photoélectrique, soit un peu plus élevée (typiquement, $0,7 \mathrm{~V}$ ), le rendement reste inférieur à $1 \%$.

\section{Longueur de diffusion par les méthodes PVS} et SPV. - Le spectre du photovoltage de surface (PVS) a été étudié pour des radiations excitatrices comprises entre $0,58 \mu \mathrm{m}$ et $0,62 \mu \mathrm{m}$ correspondant à des inverses du coefficient d'absorption compris entre $5 \mu \mathrm{m}$ et $25 \mu \mathrm{m}$ environ. L'électrode bloquante que nécessite la méthode était constituée par une lamelle de verre dont la face opposée à l'échantillon était revêtue d'une couche semi-transparente d'or. Son épaisseur $(150 \mu \mathrm{m})$ était suffisante pour qu'avec la bande passante utilisée ( $12 \AA$ ), on n'ait pas à craindre la formation d'interférences d'égal ordre chromatique. La mesure du flux incident était automatiquement corrigée de l'absorption de l'or par interposition sur le faisceau d'une lame identique à celle de l'électrode. Le signal décroît généralement dans le temps, même en absence d'éclairement. Cette décroissance, probablement due à un rééquilibre de la surface libre perturbée du matériau avec l'oxygène ambiant, est rapide dans les minutes qui suivent la préparation de la surface de l'échantillon par attaque à l'acide nitrique. Elle se ralentit ensuite et atteint environ $50 \%$ après une dizaine d'heures. Par échelonnement dans le temps des mesures, il est possible de corriger cette dérive. Des évaluations répétées ont d'ailleurs montré que la valeur de $L_{\mathrm{n}}$ trouvée ne dépend pas de façon significative du laps de temps séparant les mesures de la mise en place de l'échantillon. Moyennant ces précautions, l'alignement des points expérimentaux représentant, en fonction de $1 / \alpha$, la valeur du flux incident à signal constant est le plus souvent très bon, ce qui semble cautionner la validité de la méthode dans le cas actuel. Compte tenu d'une erreur systématique possible sur les valeurs de $\alpha$ utilisées [13] et dont l'estimation nous est inaccessible, on peut évaluer à $0,5 \mu \mathrm{m}$ l'erreur entachant, dans les meilleurs cas, la valeur de $L_{n}$. La précision peut être moins bonne dans le cas d'un rapport signal/bruit défavorable [4]. Une douzaine d'échantillons ont ainsi été étudiés. La longueur de diffusion diffère d'un échantillon à l'autre sans toutefois dépasser $8 \mu \mathrm{m}$. Il est à noter que des mesures de $L_{\mathrm{n}}$ publiées précédemment faisaient apparaître des valeurs inférieures à $2 \mu \mathrm{m}$, donc en moyenne plus faibles que celles relevées ici. Le matériau étudié alors avait été obtenu, soit par synthèse directe, soit par tirage. Les résultats présents se rapportent à des cristaux obtenus uniquement par tirage et ayant une plus faible résistivité. Il serait donc tentant d'établir une corrélation entre résistivité et longueur de diffusion. Toutefois, des tentatives faites pour la confirmer en modifiant par recuit la conductivité du matériau ont, jusqu'à présent, échoué.

Le spectre normalisé du courant photovoltaïque de court-circuit (SPV) des diodes $\mathrm{Cu} / \mathrm{Cu}_{2} \mathrm{O}$ présente un maximum entre $0,5 \mu$ et $0,57 \mu \mathrm{m}$ selon les cellules (Fig. 2). Son déclin vers les faibles longueurs d'onde est généralement attribué à la prédominance croissante des processus de recombinaison, alors que son augmentation à partir des grandes longueurs d'onde est déterminée essentiellement par la création et une collection optimale des porteurs. Nous avons étudié le début de cette dernière partie du spectre

$$
(0,58 \mu \mathrm{m}<\lambda<0,62 \mu \mathrm{m})
$$

et porté l'inverse du courant en fonction de $1 / \alpha$. Moyennant l'hypothèse d'une vitesse de recombinaison superficielle sensiblement constante et d'une contribution relativement petite des porteurs créés dans la région de barrière, on doit obtenir une droite.

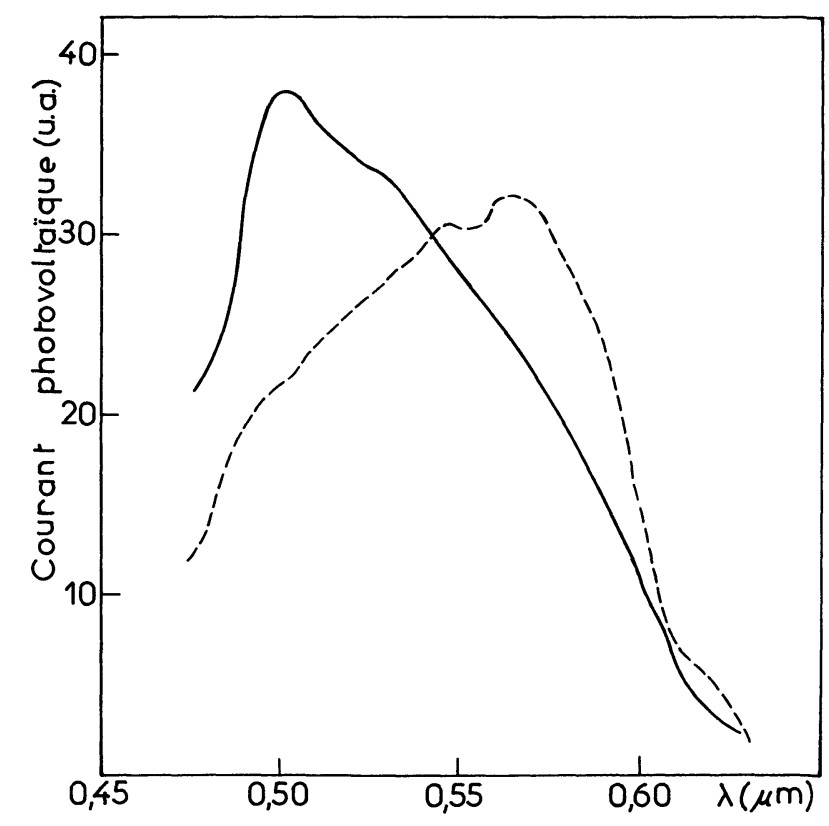

Fig. 2. - Réponse spectrale normalisée de deux diodes $\mathrm{Cu} / \mathrm{Cu}_{2} \mathrm{O}$ : a) Trait continu : $304 ; b$ ) Trait discontinu : CBO.

[Normalized spectral response of two $\mathrm{Cu} / \mathrm{Cu}_{2} \mathrm{O}$ diodes : a) Full line : $304 ; b$ ) Dashed line : CBO.] 
C'est ce qui est effectivement observé [4]. La valeur de $L_{\mathrm{n}}$ est déterminée par l'intersection de cette droite avec l'axe des abscisses. Lorsque les deux méthodes ont pu être appliquées aux mêmes échantillons, la comparaison des résultats montre une bonne concordance (Tableau I).

Tableau I. - Valeurs moyennes des longueurs de diffusion mesurées par la méthode du photovoltage de surface (PVS), par la méthode du spectre photovoltaïque (SPV) et par la méthode électronique (EBIC).

[Mean values of diffusion lengths measured by surface photovoltage method (PVS), photovoltaic spectrum method (SPV) and electron beam induced current method (EBIC).]

$\begin{array}{clcc}\text { Echantillon } & \text { PVS } & \text { SPV } & \langle\text { EBIC }\rangle \\ \text { MFC 6 } & - & - & - \\ 304 & 8 & & 2,8 \\ \text { CBO } & 3,5 & 4 & 2,8 \\ 706 & 4 & 4 & 0,2 \\ \text { CF } & 5 & 7 & \end{array}$

4. Longueur de diffusion par la méthode EBIC. La longueur de diffusion à partir du courant induit par balayage électronique (EBIC) est une méthode destructive. $\mathrm{La}$ vitesse de recombinaison superficielle des surfaces de $\mathrm{Cu}_{2} \mathrm{O}$ polies ou attaquées chimiquement étant vraisemblablement trop grande, on n'obtient un courant de diode mesurable que lorsque l'impact électronique se fait sur des facettes de clivage. Il faut donc cliver la cellule perpendiculairement à la jonction, ce qui entraîne sa destruction partielle et empêche, le plus souvent, la poursuite d'autres expériences. Le rendement de la génération des paires électron-trou ainsi que la pénétration dans le cristal des électrons incidents sont des fonctions croissantes de la tension d'accélération [10]. Nous avons utilisé habituellement des énergies de faisceau comprises entre 12 et $15 \mathrm{keV}$. D'une part, les énergies sont assez élevées pour que les paires électron-trou soient créées à des profondeurs telles que la recombinaison superficielle ne soit pas grande ; d'autre part, elles sont assez faibles pour que la zone de création soit de dimensions réduites et que le déclin spatial du courant induit obéisse à la loi $I \propto \exp \left(-x / L_{\mathrm{n}}\right)$ où $x$ est la distance du point d'impact à la jonction collectrice.

La figure 3 donne un exemple typique de la variation du courant induit, lors d'un balayage. Sa variation exponentielle est observable sur une dizaine de $\mu \mathrm{m}$, mais au lieu de se poursuivre jusqu'à l'interface $\mathrm{Cu}-\mathrm{Cu}_{2} \mathrm{O}(x=0)$, elle cesse à quelques $\mu \mathrm{m}$ du métal et l'on observe une chute du courant. Nous avons procédé à plusieurs groupes de balayages sur une même facette de clivage, les trois à six balayages de chaque groupe étant distants les uns des autres d'une

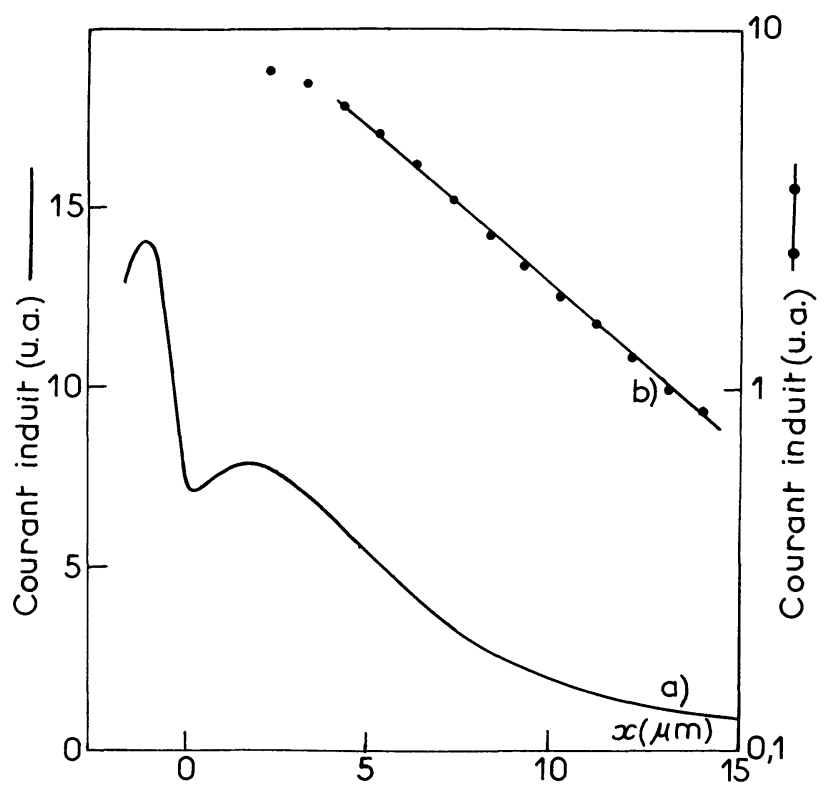

Fig. 3. - Courant induit par balayage électronique en fonction de la distance du point d'impact à la jonction $(x=0)$. a) Echelle linéaire ; $b$ ) Echelle semi-logarithmique.

[Electron beam induced current versus scan distance on a $\mathrm{Cu} / \mathrm{Cu}_{2} \mathrm{O}$ junction $(x=0)$. a) Linear plot; b) Semi-logarithmic plot.]

dizaine de $\mu \mathrm{m}$ et les groupes étant répartis le long de la facette c'est-à-dire sur une distance de 1 à 2 mm suivant le cas. Les longueurs de diffusion déterminées à partir de la portion des courbes où le courant varie exponentiellement présentent une dispersion assez marquée, tant à l'intérieur des groupes que d'un groupe à l'autre. La comparaison des lignes et des colonnes du tableau II montre qu'il s'agit d'inhomogénéités à courte portée. La confrontation des résultats obtenus par cette méthode et par les méthodes PVS et SPV (cf. Tableau I), bien que ne portant que sur trois échantillons indique que les longueurs de diffusion mesurées par la méthode EBIC sont plus petites.

Tableau II. - Valeurs de la longueur de diffusion relevées sur des diodes $\mathrm{Cu} / \mathrm{Cu}_{2} \mathrm{O}$ par la méthode $\mathrm{EBIC}$, en différents endroits de la surface.

[Values of the diffusion length measured on $\mathrm{Cu} / \mathrm{Cu}_{2} \mathrm{O}$ diodes by EBIC method on various points of the surface.]

\begin{tabular}{cccccccc} 
Echantillon & Groupe & \multicolumn{7}{c}{$L_{\mathrm{n}}$} \\
- & I & 1,8 & 2,5 & 5,0 & & & \\
MFC 6 & II & 2,1 & 2,0 & 2,1 & 1,8 & 2,2 & 3,6 \\
& III & 4,5 & 2,8 & 2,5 & 2,5 & 2,6 & 3,1 \\
& I & 2,0 & 2,5 & 5,1 & 2,9 & & \\
304 & II & 3,0 & 3,0 & 2,0 & 2,2 & & \\
& III & 2,3 & 4,4 & 2,1 & & & \\
& I & 0,1 & 0,1 & 0,2 & & & \\
CBO & II & 0,3 & & & & & \\
& III & 0,3 & 0,2 & 0,2 & & &
\end{tabular}


5. Le problème de la jonction. - La photo de la figure 4 a été obtenue au microscope électronique à balayage, comme il a été indiqué en début d'article. La plus ou moins grande densité optique correspond à un courant plus ou moins intense résultant de l'impact électronique au point considéré. Outre l'aspect peu homogène de la bande sombre marquant la région dont un pourcentage appréciable des charges induites sont collectées, on remarque le liséré clair qui sépare cette bande de l'électrode de $\mathrm{Cu}$. Il traduit la décroissance du courant notée sur la courbe de la figure. La largeur de cette zone, variable d'un échantillon à l'autre, est le plus souvent de $1 \mu \mathrm{m}$ à $3 \mu \mathrm{m}$. Nous n'avons observé son absence que dans un cas (échantillon CBO, Tableau II). Mais comme la longueur de diffusion mesurée par la méthode EBIC est alors très petite (de l'ordre de $0,1 \mu \mathrm{m}$ ) et le courant induit très faible, il se pourrait que l'absence d'une apparence de liséré ne résulte, en fait, que d'une largeur plus grande de la couche semi-passive. Il semble donc que cette couche dont, à notre connaissance, la présence n'a jamais été signalée, soit caractéristique des jonctions frontales $\mathrm{Cu}-\mathrm{Cu}_{2} \mathrm{O}$. Avec les cellules dorsales obtenues en oxydant partiellement le cuivre, une anomalie du courant induit, mais d'une autre nature semble-t-il, a également été observée [14].

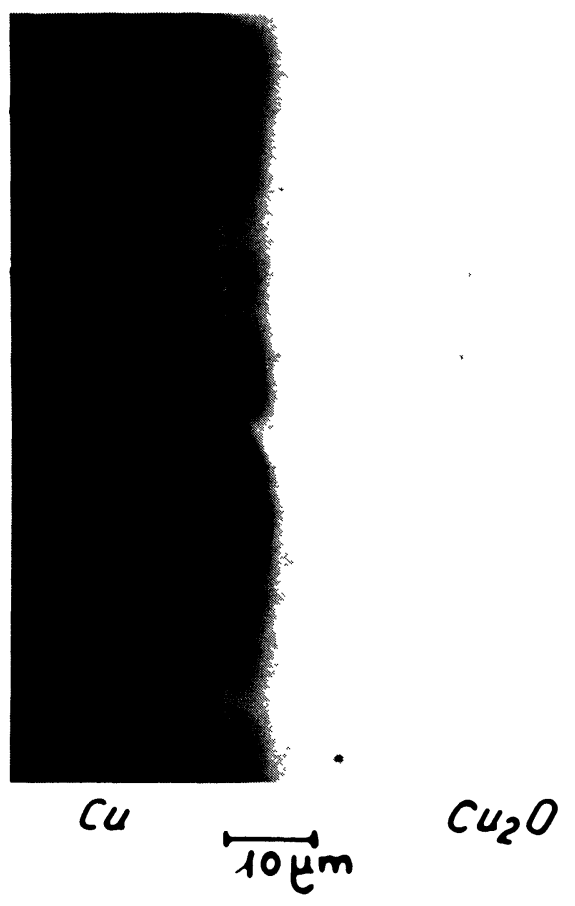

Fig. 4. - Image « EBIC » typique d'une jonction $\mathrm{Cu} / \mathrm{Cu}_{2} \mathrm{O}$.

[Typical « EBIC » image of a $\mathrm{Cu} / \mathrm{Cu}_{2} \mathrm{O}$ junction.]

Parmi les hypothèses qu'on peut avancer pour expliquer l'existence de cette couche, il faut écarter d'emblée celle d'une moindre création de paires liée à une plus faible absorption des électrons incidents : l'examen conjoint des variations spatiales du courant

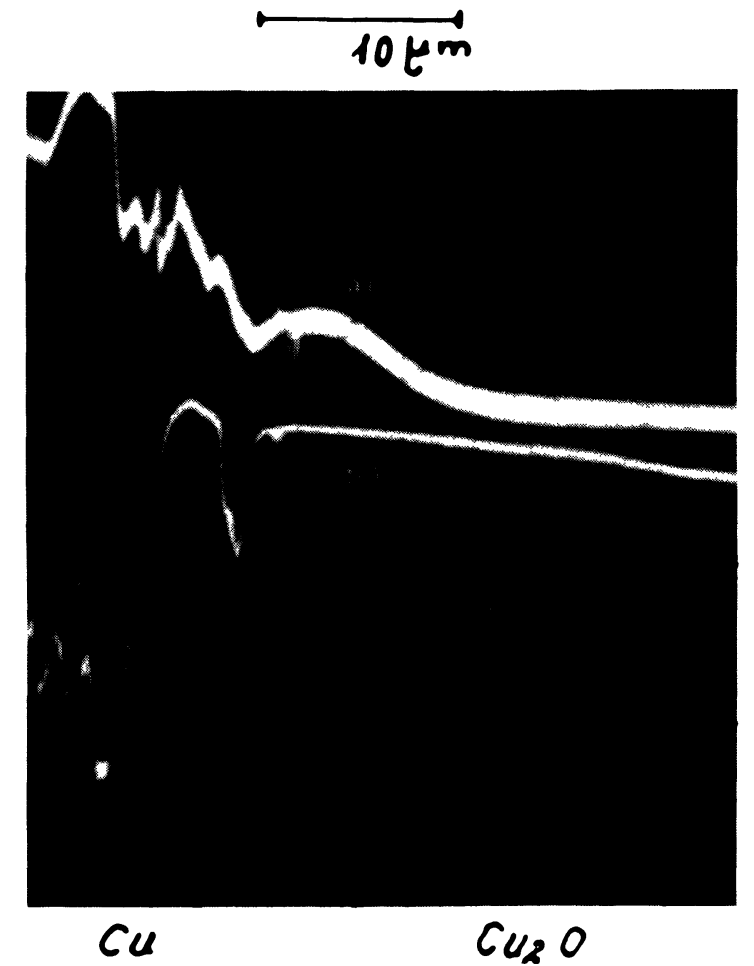

Fig. 5. - Courant induit (a) et courant des électrons secondaires (b) correspondant.

[Induced current $(a)$ and corresponding secondary electron current $(b)$.]

induit et du courant rétrodiffusé (Fig. 5) montre que ce dernier ne présente aucune anomalie au voisinage du cuivre.

Par contre, il faut retenir l'hypothèse d'une couche superficielle d'épaisseur finie pré-existant au dépôt de l'électrode dans laquelle les porteurs minoritaires créés auraient une durée de vie bien moindre que dans le volume. Les études des résistivités de surface et de volume [11] ont abondamment prouvé l'existence d'une telle couche et les mesures d'effet photomagnétoélectrique [15] (faites, il est vrai, à la température de l'azote liquide) ont montré que la durée de vie des électrons décroissait fortement au fur et à mesure que la radiation excitatrice était principalement absorbée dans des strates de plus en plus voisines de la surface de l'échantillon. Bien qu'il soit difficile d'évaluer l'épaisseur exacte de cette couche de surface, des valeurs de l'ordre $\mathrm{du} \mu \mathrm{m}$ sont très vraisemblables.

Des expériences en cours montrent que les performances des cellules préparées sous vide se dégradent progressivement au contact de l'atmosphère. Inversement, on améliore, par recuit sous vide, les performances de cellules ayant séjourné dans l'atmosphère ambiant. Ceci semble indiquer que l'existence de la couche semi-passive est liée à un enrichissement en oxygène de la surface.

Pour étudier l'influence de la zone semi-passive sur le comportement des cellules, nous avons assimilé le cas concret au schéma simple d'une barrière séparée 
du matériau émetteur par une région d'épaisseur $\xi_{0} L_{\mathrm{n}}$. Nous avons d'abord examiné si notre hypothèse expliquait la petitesse des densités de courant de court-circuit $J_{\mathrm{cc}}$. Si l'on néglige toute autre recombinaison superficielle ou effet interfacial, le rapport du $J_{\mathrm{cc}}$ observé au $J_{\mathrm{cc}}^{(0)}$ d'une cellule idéale est donné par l'expression :

$$
R=\frac{J_{\mathrm{cc}}}{J_{\mathrm{cc}}^{(0)}}=\frac{\int \psi(\lambda) r(\lambda) \mathrm{d} \lambda}{\int \psi(\lambda) \mathrm{d} \lambda}
$$

avec

$\psi(\lambda)=\frac{\varphi(\lambda)}{1+K(\lambda)} \quad$ et $\quad r(\lambda)=\exp \left[-\left(1+\frac{1}{K(\lambda)}\right)\right] \xi_{0}$

$\varphi(\lambda)$ est la densité spectrale des photons pour le type d'éclairement utilisé et la grandeur sans dimension $K(\lambda)$ est l'inverse du coefficient d'absorption mesuré en prenant $L_{\mathrm{n}}$ pour unité. Pour un éclairement de type AM $1, \xi_{0}=2 / 3$ et $L_{\mathrm{n}}=3 \mu \mathrm{m}$ (ce qui correspond à une situation concrète moyenne), nous trouvons une valeur de $R$ de $15 \%$ environ. En fait, si l'on pouvait prendre en compte les inhomogénéités de la collection qui réduisent la surface efficace de la jonction, l'atténuation serait encore plus forte. Les évaluations faites par Wang et al. [6] de la densité de courant $J_{\mathbf{c c}}^{(0)}$ montrent qu'avec $L_{\mathrm{n}}=3 \mu \mathrm{m}$, on obtient une valeur limite un peu inférieure à $10 \mathrm{~mA} / \mathrm{cm}^{2}$. Notre résultat indique, par conséquent, que le $J_{\mathrm{cc}}$ de la plupart de nos cellules ne devrait pas dépasser le $\mathrm{mA} / \mathrm{cm}^{2}$, conclusion qui rejoint effectivement l'expérience.

Nous avons également évalué le déplacement en longueurs d'onde du pic de la réponse spectrale lorsqu'on augmente l'épaisseur de la couche passive. La position de ce pic est donnée par le maximum de l'expression :

$$
J_{\mathrm{cc}}\left(\xi_{0}+\Delta \xi_{0} ; \lambda\right)=J_{\mathrm{cc}}\left(\xi_{0} ; \lambda\right) \exp -\left(1+\frac{1}{K(\lambda)}\right) \Delta \xi_{0}
$$

où $J_{\mathrm{cc}}\left(\xi_{0}+\Delta \xi_{0} ; \lambda\right)$ et $J_{\mathrm{cc}}\left(\xi_{0} ; \lambda\right)$ sont respectivement les réponses spectrales pour une couche passive d'épaisseur $\left(\xi_{0}+\Delta \xi_{0}\right) L_{\mathrm{n}}$ et $\xi_{0} L_{\mathrm{n}}$. On voit aisément qu'une augmentation de $\xi_{0}$ déplace le pic vers les grandes longueurs d'onde. Aussi, parmi les spectres expérimentaux, avons-nous choisi celui dont le maximum se situe aux plus faibles longueurs d'onde (5000 ̊) et avons-nous étudié ce qu'il en adviendrait si l'épaisseur de la couche augmentait. Le résultat de ces calculs (avec $L_{\mathrm{n}}=3 \mu \mathrm{m}$ ) est illustré par la figure 6 . On remarque que le pic peut se déplacer sur environ $1000 \AA$ et que son flanc, côté grandes longueurs d'onde, devient de plus en plus abrupt lorsque $\xi_{0}$ croît. L'examen de la réponse spectrale de la cellule $\mathrm{CBO}$, cellule dans laquelle tous les résultats expérimentaux semblent indiquer la présence d'une couche

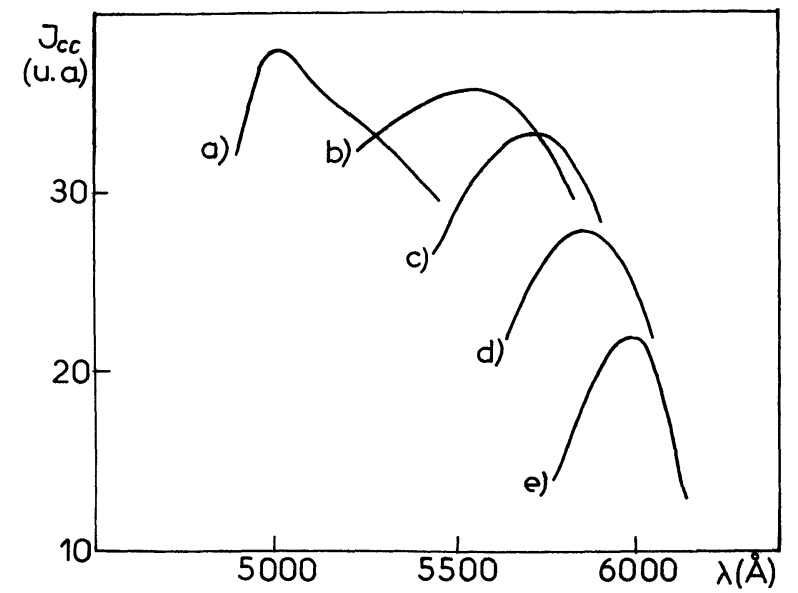

Fig. 6. - Déplacement du maximum de la réponse spectrale lorsque l'épaisseur de la couche passive augmente de $L_{\mathrm{n}} \Delta \xi_{0}$. Calculs pour $L_{\mathrm{n}}=3 \mu \mathrm{m}$. a) Réponse spectrale originale; $\left.b\right) \Delta \xi_{0}=1 / 4(\mathrm{X} 2,5)$; c) $\left.\left.\Delta \xi_{0}=1 / 2(\mathrm{X} 4) ; d\right) \Delta \xi_{0}=1(\mathrm{X} 8) ; e\right) \Delta \xi_{0}=2(\mathrm{X} 25)$.

[Shift of the maximum of the spectral response when the thickness of the passive layer increases by an amount $L_{\mathrm{n}} \Delta \xi_{0}$. Calculations for $L_{\mathrm{n}}=3 \mu \mathrm{m}$. a) Initial spectral response ; b) $\Delta \xi_{0}=1 / 4(\mathrm{X} 2,5)$; c) $\left.\left.\Delta \xi_{0}=1 / 2(\mathrm{X} 4) ; d\right) \Delta \xi_{0}=1(\mathrm{X} 8) ; e\right) \Delta \xi_{0}=2(\mathrm{X} 25)$.]

passive épaisse, confirme semi-quantitativement et qualitativement le comportement précédent : le maximum est déplacé de $700 \AA \AA$ et la montée du courant est nettement plus rapide que dans les spectres ayant leur pic vers $5000 \AA$.

Il reste à examiner l'incidence de notre hypothèse sur l'interprétation du résultat des mesures de $L_{\mathrm{n}}$. Avec la méthode EBIC, elle se manifeste par une chute du rendement au voisinage de l'électrode et l'apparition, sur l'image électronique, du liséré clair que nous avons signalé.

Toutefois, lorsque la couche est relativement épaisse, le courant induit par la génération de paires au sein du matériau devient très faible et se confond avec le bruit : l'image électronique prend l'aspect uniforme observé avec l'échantillon CBO.

Dans la méthode PVS, le flux lumineux à photovoltage de surface constant $[\varphi(\lambda)]$ devient proportionnel à :

$$
\left(1+\frac{1}{\alpha L_{\mathrm{n}}}\right) \exp \left(\alpha \xi_{0} L_{\mathrm{n}}\right) \text {. }
$$

La figure 7 montre l'évolution de $[\varphi]_{\mathrm{v}}=f(1 / \alpha)$ calculée pour différentes épaisseurs relatives $\xi_{0}$ de la couche. L'expression ci-dessus se comporte sensiblement comme $1+\xi_{0}+\frac{1}{\alpha L_{n}}$ dès que $1 / \alpha$ est de l'ordre de quelques $\xi_{0} L_{n}$, mais présente, par contre, un caractère non linéaire en $1 / \alpha$ pour les faibles valeurs de cette variable. De fait, sur les courbes expérimentales, on note que le premier point $(1 / \alpha \simeq 5 \mu \mathrm{m}$ pour $\lambda=0,58 \mu \mathrm{m})$ se trouve fréquemment au-dessus de la droite joignant les autres points. D'autre part, la valeur de $1 / \alpha$ extrapolée à $[\varphi]_{\mathrm{v}}=0$ est $-L_{\mathrm{n}}\left(1+\xi_{0}\right)$, ce qui expliquerait que la longueur de diffusion 


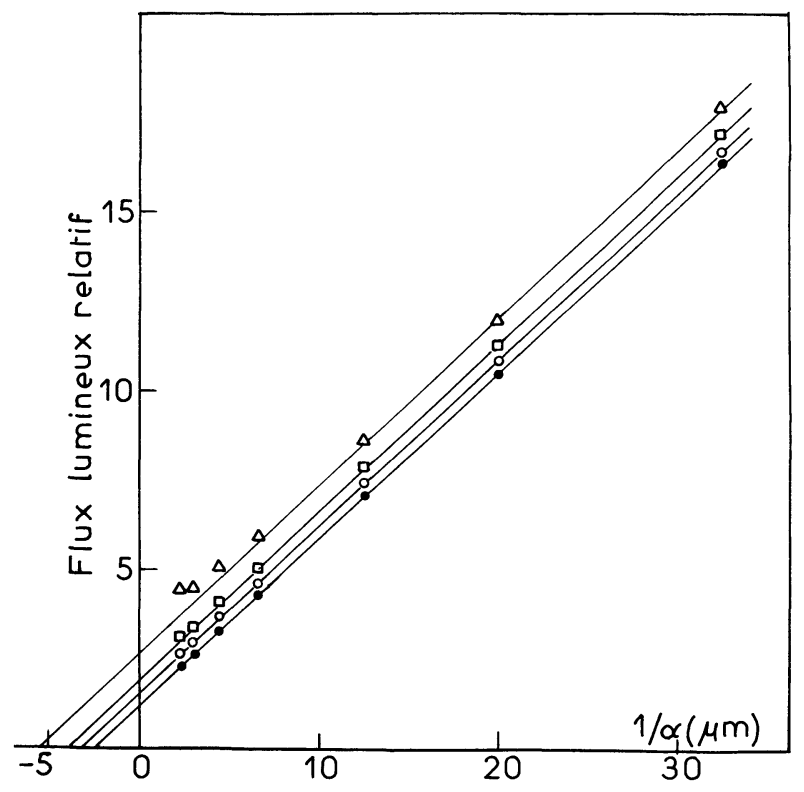

Fig. 7. - Determination de $L_{\mathrm{n}}$ par la méthode PVS pour des couches passives de différentes épaisseurs. $\bullet \xi_{0}=0 ; \circ \xi_{0}=1 / 4$; $\square \xi_{0}=1 / 2 ; \Delta \xi_{0}=1$.

[Determination of $L_{\mathrm{n}}$ by the PVS method for passive layers of variable thickness. $\bullet \xi_{0}=0 ; \bigcirc \quad \xi_{0}=1 / 4 ; \square \xi_{0}=1 / 2 ; \Delta \xi_{0}=1$.]

apparente mesurée par cette méthode soit supérieure à celle déduite des mesures EBIC. Les mêmes remarques s'appliquent à l'interprétation des résultats obtenus par la méthode SPV.

6. Conclusion. - Les mesures de la longueur de diffusion effectuées par les trois méthodes PVS, SPV et EBIC concordent en ordre de grandeur. $L_{n}$ ne dépasse pas ep moyenne quelques $\mu \mathrm{m}$. Toutefois, les fluctuations locales révélées par microscopie électronique à balayage indiquent que des valeurs approchant $10 \mu \mathrm{m}$ ne sont pas incompatibles avec la nature du matériau. Nous ne sommes pas parvenus à atteindre ce résultat dans nos essais préliminaires de traitement du matériau dans le but d'améliorer la longueur de diffusion. L'effort mérite-t-il d'être poursuivi ? Avec de telles longueurs de diffusion, on peut évaluer [6] à environ $13 \mathrm{~mA} / \mathrm{cm}^{2}$ la densité de courant que pourrait fournir une cellule idéale. Or, on constate que beaucoup des cellules réelles ne délivrent même pas le vingtième du courant optimal. Dans ces conditions et avec un $V_{\text {co }}$ de $0,5 \mathrm{~V}$ et un facteur de remplissage de 0,5 (meilleures valeurs relevées), le rendement resterait inférieur à $0,25 \%$. Comme $J_{\text {cc }}$ tend ensuite lentement à la saturation lorsque croît $L_{\mathrm{n}}$, une augmentation supplémentaire de $L_{\mathrm{n}}$ n'entraînerait qu'un gain négligeable.

Il convient donc d'améliorer la qualité de la collection. Nous avons montré que la présence d'une couche superficielle, d'épaisseur comparable à $L_{\mathrm{n}}$ où les porteurs créés auraient une très faible durée de vie, explique très bien le comportement des cellules (faibles $J_{\mathrm{cc}}$, allure de la réponse spectrale, écart des valeurs de $L_{\mathrm{n}}$ mesurées par la méthode EBIC d'une part et par les méthodes PVS et SPV d'autre part) et est en accord avec un ensemble d'observations antérieures.

Les expériences menées lors de nos études de la conductibilité de $\mathrm{Cu}_{2} \mathrm{O}$ indiquent qu'il n'est pas irréaliste d'espérer développer une technique qui fasse disparaître cette couche et, du même coup, réduise les inhomogénéités de la région de jonction. La recombinaison interfaciale seule limiterait alors la densité de courant. Même en ignorant cette limitation et en supposant une amélioration du facteur de remplissage, le rendement ne dépasserait pas $4 \%$. On ne pourrait, dès lors, atteindre un accroissement substantiel de celui-ci que d'une augmentation de la hauteur de barrière. Or, il semble bien que la tension en circuit ouvert soit limitée à $0,5 \mathrm{~V}$ environ par la nature même du contact $\mathrm{Cu} / \mathrm{Cu}_{2} \mathrm{O}$. La substitution au cuivre d'un métal de plus faible potentiel d'extraction a été décevante [3] : les travaux de Olsen et al. [2] tendent à montrer que, dans ce cas, la diffusion du métal et la réduction de $\mathrm{Cu}_{2} \mathrm{O}$ par celui-ci ramènent le contact à une jonction $\mathrm{Cu} / \mathrm{Cu}_{2} \mathrm{O}$. La conversion de l'énergie solaire, avec un rendement économiquement acceptable, au moyen de $\mathrm{Cu}_{2} \mathrm{O}$, ne paraît donc être réalisable qu'en utilisant des hétérojonctions ou encore des jonctions de type MIS, à condition, d'une part qu'on arrive à neutraliser la couche de surface et, d'autre part qu'on trouve un partenaire ne réagissant pas avec $\mathrm{Cu}_{2} \mathrm{O}$.

Remerciements. - Les mesures au microscope électronique ont été faites au Laboratoire de Microscopie Electronique de Montpellier. Nous remercions Monsieur Bresse de l'aide qu'il nous a prodiguée.

Nous remercions le Dr C. Schwab pour les discussions que nous avons eues avec lui au cours de cette étude. 


\section{Bibliographie}

[1] Hérion, J., Natsch, B., Niekisch, E. A., Scharl, G., Photovoltaic Solar Energy Conf., Berlin (1979), à paraître.

[2] Olsen, L. C., Bohara, R. C., Urie, M. W., Appl. Phys. Lett. 34 (1979) 47.

[3] Trivich, D., Wang, Y. W., Komp, R. J., Ho, F., 12th IEEE Photovoltaic Specialists Conference Proc., Bâton Rouge (1976).

[4] Tapiéro, M., Noguet, C., Zielinger, J. P., Schwab, C., Pierrat, D., Revue Phys. Appl. 14 (1979) 231.

[5] Loferski, J. J., 3rd Photovoltaic Specialists Conference (1963) 667.

[6] Wang, E. Y., Trivich, D., Sawalka, H., Thomas, G., Proc. of Comples International Conf., Dharan, Saudi Arabia, Heliotechnique and Development 1 (1976) 643.

[7] Trivich, D., WaNG, E. Y., Komp, R. J., KaKaR, A. S., 13th IEEE Photovoltaic Specialists Conference (1978).

[8] Goodman, A. M., J. Appl. Phys. 32 (1961) 2550.
[9] Quilliet, A., Gosar, P., J. Physique Rad. 21 (1960) 575.

[10] Microanalyse et Microscopie à Balayage, Les Editions de Physique (Orsay)

[11] Zielinger, J. P., Tapiero, M., Noguet, C., Ann. Phys. 7 (1972) 95

Tapiero, M., Zielinger, J. P., Noguet, C., Phys. Status Solidi (a) 33 (1976) 155.

[12] Tapiero, M., Zielinger, J. P., Noguet, C., Phys. Status Solidi (a) 12 (1972) 517.

[13] Pastrnyak, I., Sov. Phys. Sol. State 1 (1959) 888.

[14] Mizuguel, J., Japan J. Appl. Phys. 15 (1976) 907.

[15] Zielinger, J. P., Thèse, Strasbourg (1969).

[16] Fahrenbruch, A. L., Aranovich, J., Courrages, F., Yin, S. Y. and BubE, R. H., Photovoltaic Solar Energy Conference, Proceedings of the International Conference, Luxembourg (sept. 1977). 